\title{
Discrete Modal Decomposition for surface appearance modelling and rendering
}

\author{
Gilles Pitard ${ }^{a}$, Gaëtan Le Goïc ${ }^{b}$, Hugues Favrelière ${ }^{a}$, Serge Samper ${ }^{a, c}$, Simon-Frédéric Desage, ${ }^{a}$ \\ Maurice Pillet ${ }^{a}$ \\ ${ }^{a}$ Laboratoire Systèmes et Matériaux pour la Mécatronique, SYMME EA 4144, Université de \\ Savoie, Annecy, France; \\ ${ }^{b}$ Laboratoire Electronique, Informatique et Image, LE2I UMR CNRS 6306, Université de \\ Bourgogne, Auxerre, France; \\ ${ }^{c}$ Laboratoire de Recherche en Mécanique Appliquée, LARMAUR - ERL CNRS 6274, \\ Université de Rennes, Rennes, France;
}

\begin{abstract}
Controlling surface appearance has become essential in the supplier/customer relationship. In this context, many industries have implemented new methods to improve the sensory inspection, particularly in terms of variability. A trend is to develop both hardware and methods for moving towards the automation of appearance inspection and analysis. If devices inspired from dimensional control solutions generally allow to identify defects far apart the expected quality of products, it do not allow to quantify finely appearance anomalies, and decide on their acceptance.

To adress this issue, new methods devoted to appearance modelling and rendering have been implemented, such as the Reflectance Transformation Imaging (RTI) technique. By varying the illumination positions, the RTI technique aims at enriching the classical information conveyed by images. Thus each pixel is described by a set of values rather than one value classically; each value corresponding to a specific illumination position. This set of values could be interpolated or approximated by a continuous model (function), associated to the reflectance of the pixel, generally based on a second order polynomial (namely, Polynomial Texture Mapping Technique). This paper presents a new approach to evaluate this information from RTI acquisitions. A modal projection based on dynamics (Discrete Modal Decomposition) is used to estimate surface reflectance on each measurement point. After presenting the acquisition device, an application on an industrial surface is proposed in order to validate the approach, and compare it to the more classical polynomial transformation. Results show that the proposed projection basis not only provides closer assessment of surface reflectance (modelling) but also yields to a more realistic rendering.
\end{abstract}

Keywords: Discrete Modal Decomposition, Reflectance Transformation Imaging, Surface appearance, BRDF

\section{INTRODUCTION}

In many industrial fields, such as micro-mechanics, biomedicine, horology, surface quality is measured visually by human observation of reflected images on the surface, viewed under controlled lighting conditions in order to identify potential defects. The sensory evaluation is often hand-operated, quality controllers vary viewing and light incidence angles on the surface to ensure that they have covered a wide range of configurations. In order to limit the risk of undetected appearance defects, specific configurations are sought (Figure 1): black light effect, white light effect and mirror effect. Based on the observation of this current practice, our approach consists in analysing the interaction of surfaces with their light environment thanks to an estimate of the biangular components of the reflectance function ${ }^{1-3}$ which quantifies the light reflecting off an object. To adress the modelling of reflectance, three approachs differ in complexity and accuracy to approximate the Bidirectional Reflectance Distribution Function (BRDF) which is one of the most complete physical reflection model:

Corresponding author, Gilles.Pitard@univ-savoie.fr 

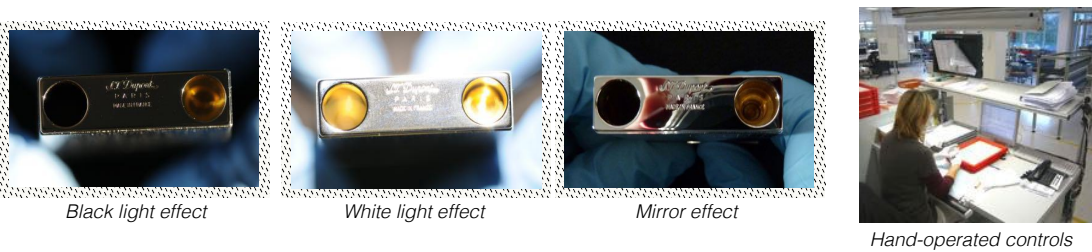

Figure 1. Sensory effects obtained in different light configurations of a polished lighter (bottom view) by quality controllers

- model-based rendering methods consist in the parametrization on analytical BRDF models. This approach is not suitable to adress the appearance quality control due to the complexity of surface textures and light behavior make it difficult to apply to the real materials.

- measuring the complete Bidirectional Reflectance Distribution Function. A dense reflectance sample at a single surface point is generally acquired by a gonioreflectometer. ${ }^{4-6}$ This method provides a complete description of the interaction light/surface, but will lead to the generation of huge amounts of data and the cost of acquiring data make it incompatible with an application in the context of surface inspection, especially when applied to finding defects.

- image-based rendering methods rely on the acquisition of photometric data (only the direction of illumination varies between images), similar approach as known as $\boldsymbol{R}$ eflectance Transformation Imaging techniques. ${ }^{7,8}$

Thus, our approach is based on the RTI techniques which can accurately model a surface appearance without needing knowledge of its materials or geometrical properties. Several RTI-based techniques have been developped for better measured approximation to reflectance field that uses a set of images - taken from a camera in a fixed position - of the object surface illuminated from different light directions.

The RTI approach and a sphere capture system are presented in Section 2. After presenting the usual RTI methods (Section 2.3), a new modelling technique of angular components of local reflectance from the set of images based on the Discrete Modale Decomposition is presented in Section 3. A comparison with Polynomial Texutre Mappings (PTM) and Hemispherical Harmonics (HSH) is applied to high value-added industrial surfaces in Section 4. It appears that the DMD technique allows us to aid in quantifying particular aspects of surface appearance by preserving spatially local luminance variations in the reconstructed images.

\section{REFLECTANCE TRANSFORMATION IMAGING}

\subsection{RTI approach}

A proportional relationship exists between the gray level $G$ of an image and the luminance $L\left(\overrightarrow{u_{c}}\right)$ along the optical axis $\overrightarrow{u_{c}}$ even if the estimate is biased in presence of cast shadows or overexposed regions. As similar formulations used in numerous papers about Shape-From-Shading, the gray level of an image can be expressed as follows: ${ }^{9}$

$$
G=k \times g \quad \text { with } \quad g=L\left(\overrightarrow{u_{c}}\right)
$$

where $k$ is the factor of proportionality, and $g$ is the normalized gray level. A camera can consenquently be used to make measurements of the luminance of associated pixel values. The RTI principe can divide in 3 steps:

1. Acquisition: The RTI acquisition device provide for each pixel a set of corresponding luminance values for different angles of illumination. The set of intensities at each pixel location are captured by a fixed camera at position $\left(\theta_{v}, \phi_{v}\right)$ under different light directions $\left(\theta_{i}, \phi_{i}\right)$.

2. Modelling: The bidirectional reflectance function BRDF is approximated from the discrete luminance values to model a continuous information. The surface reflectance is approximated by an RTI method in which different approaches are detailed from the Section 2.2. 


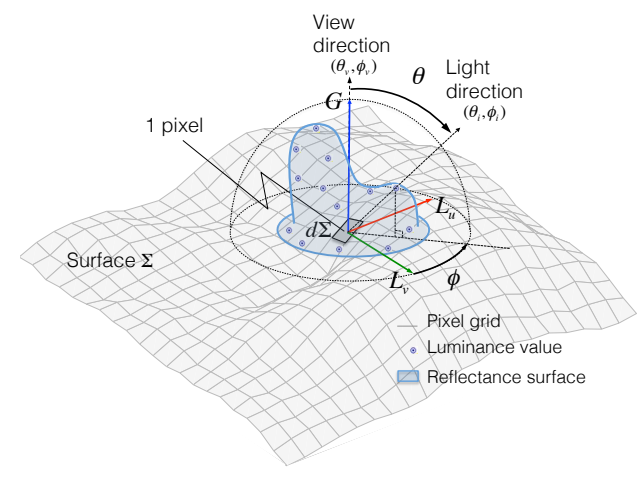

Figure 2. Light direction parametrization $l_{u}, l_{v}$

3. Rendering: The model enables to compute a reflectance function that can be used to interactively display images under novel lighting conditions varying a virtual source light controlled by a user to reveal surface phenomena.

The surface reflectance is modelled and represented in the $U V$ texture coordinate system ${ }^{10}$ (Figure 2) where $\left(l_{u}, l_{v}\right)$ are the projections of the normalized light vector onto the local basis $\left(L_{u}, L_{v}\right)$ of a particular pixel at the spatial coordinates $(u, v)$ in the studied surface. The descriptors of HSH and DMD techniques are expressed as functions of angles for azimuth $\phi$ and co-latitude $\theta$ by the following change of variables:

$$
\phi=\arctan 2\left(l_{u}, l_{v}\right) \quad \text { and } \quad \theta=\arccos \left(\sqrt{1-l_{u}^{2}-l_{v}^{2}}\right)
$$

\subsection{Image acquisition}

Figure 3 shows the steps involved in implementing the RTI techniques which require the acquisition of multiple images of a surface with a fixed camera under varying lighting conditions. A system with multiple cameras positioned around a dark sphere was developed. It enables the acquisition of surfaces-of-revolution or freeform surfaces which requires several viewing angles, such as spherical roller bearings (aeronautics), femoral heads of hip implant (medical) or rings (jewellery) because visual coverage of the entire surface can be an issue using a single scene description. The multi-camera system also opens up the creation enriching new methods for 3D reconstruction, surface analysis based on curvatures and slopes, or even measuring transmittance properties of transparent materials. In the present paper, we focus on the upper dome of our spherical structure using only a single camera (cam1) for gathering and processing reflectance transformation imaging. The object is positioned on a glass at the center of the sphere (65 cm diameter). 56 LED light sources are homogenously distributed over the upper hemisphere and above the inspected surface.

\subsection{Existing modelling techniques}

\subsubsection{Polynomial Texture Mapping}

Polynomial Texture Mappings was originally developped by Tom Malzbender and Al. ${ }^{10-16}$ The classical PTM model consists in approximating the BRDF from the discrete values of luminance obtained by the device, via polynomial regression. For each pixel, we can seek a polynomial regression 6-vectors of coefficients $\left(a_{0}-a_{5}\right)$ of the quadratic surface defined by the second degree polynomial equation 3. The regressing is carried out using Least Squares formulation (Eq. 4) and solved using Singular Value Decomposition (SVD):

$$
\begin{gathered}
L\left(l_{u}, l_{v}\right)=a_{0}+a_{1} l_{u}+a_{2} l_{v}+a_{3} l_{u} l_{v}+a_{4} l_{u}^{2}+a_{5} l_{v}^{2} \\
{\left[\begin{array}{llllll}
l_{u 0}^{2} & l_{v 0}^{2} & l_{u 0} l_{v 0} & l_{u 0} & l_{v 0} & 1 \\
l_{u 1}^{2} & l_{v 1}^{2} & l_{u 1} l_{v 1} & l_{u 1} & l_{v 1} & 1 \\
\cdot & \cdot & \cdot & \cdot & \cdot & \cdot \\
\cdot & \cdot & \cdot & \cdot & \cdot & \cdot \\
\cdot & \cdot & \cdot & \cdot & \cdot & \cdot \\
l_{u N}^{2} & l_{v N}^{2} & l_{u N} l_{v N} & l_{u N} & l_{v N} & 1
\end{array}\right]\left[\begin{array}{l}
a_{5} \\
a_{4} \\
a_{3} \\
a_{2} \\
a_{1} \\
a_{0}
\end{array}\right]=\left[\begin{array}{l}
L_{0} \\
L_{1} \\
\cdot \\
\cdot \\
\cdot \\
L_{N}
\end{array}\right]}
\end{gathered}
$$



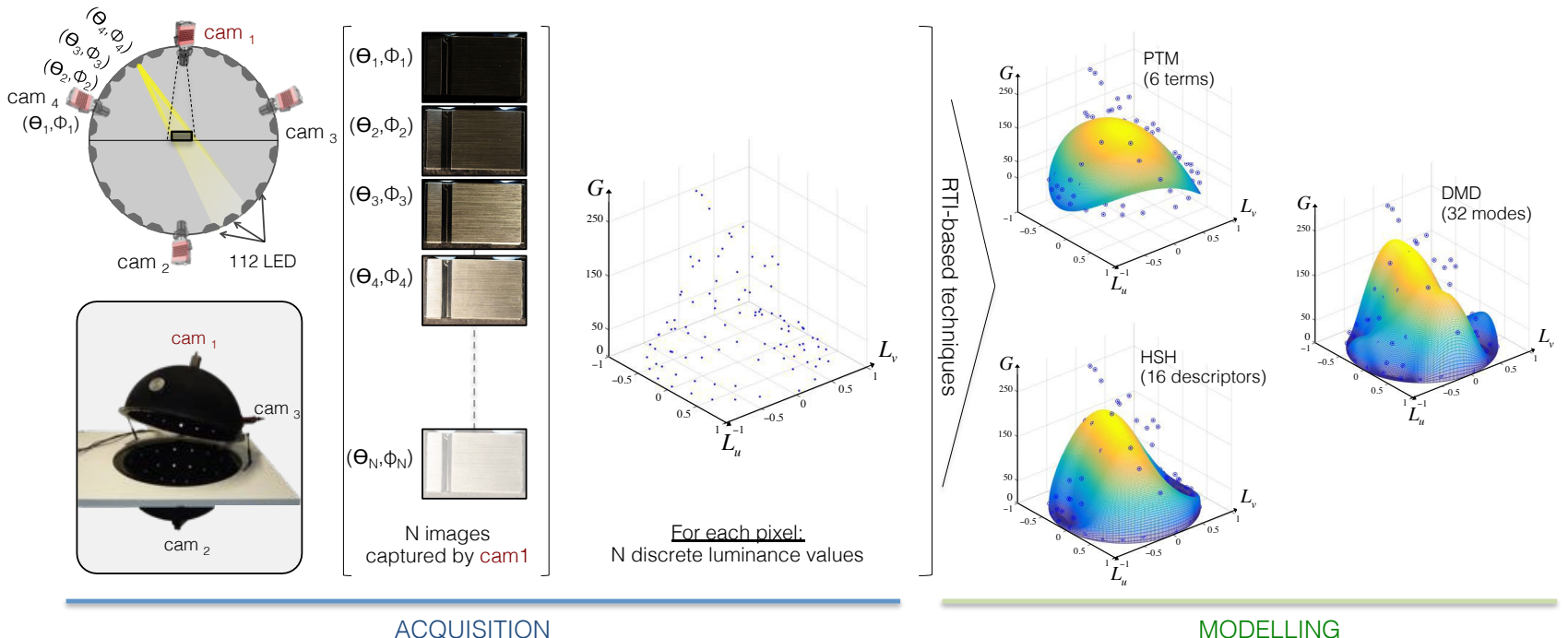

Figure 3. Diagram of the automatic acquisition of $\mathrm{N}$ images for gathering and processing Reflectance Transformation Imaging

Hence, we can interpolate and relight in order to simulate the rendering of a surface under any chosen light direction. PTM descriptors are basic shapes and they provide limited estimation about the overall shape of reflectance field (ie. high-frequency components removed). PTM tends to smooth out the fine details of the surface where sharp shadow boundaries and highly specular surfaces occur.

\subsubsection{Hemispherical Harmonics}

HemiSpherical Harmonics ${ }^{17,18}$ is an evolution of the PTM technique. This method consists in using a more appropriate projection into a set of basis functions, based on hemispherical harmonics whose shapes are close to reflectance field. HSH have been applied in several topics in computer graphics such as the modelling of BRDFs, irradiance environment maps or invariant object recognition. The hemispherical harmonics $H_{l}^{m}$ are derived from Spherical Harmonic functions (SH) using shifted associated Legendre Polynomials (Eq. 5) to map these functions to a basis set defined only over the upper hemisphere (Eq. 6):

$$
\tilde{P}_{l}^{m}(\cos \theta)=P_{l}^{m}(2 \cos \theta-1) \quad \text { and } \quad \theta \in\left[0, \frac{\pi}{2}\right]
$$

where Legendre polynomials $P_{l}^{m}$ can be computed by applying recurrence relations. ${ }^{18}$

$$
H_{l}^{m}(\theta, \phi)=\left\{\begin{array}{cc}
\sqrt{2} \tilde{K}_{l}^{m} \cos (m \phi) \tilde{P}_{l}^{m}(\cos \theta) & \text { if } m>0 \\
\sqrt{2} \tilde{K}_{l}^{m} \sin (-m \phi) \tilde{P}_{l}^{-m}(\cos \theta) & \text { if } m<0 \\
\tilde{K}_{l}^{0} \tilde{P}_{l}^{0}(\cos \theta) & \text { if } m=0
\end{array}\right.
$$

where $K_{l}^{m}$ are the hemispherical normalization factors:

$$
K_{l}^{m}=\sqrt{\frac{(2 l+1)(l-|m|)}{2 \pi(l+|m|)}}
$$

Any surface reflectance function can be decomposed into a $\mathrm{HSH}$ series ${ }^{8,19-22}$ of varying order 1 and degree $\mathrm{m}$ and the decomposition residual:

$$
f_{\left(\theta_{v}, \phi_{v}\right)}\left(\theta_{i}, \phi_{i}\right)=\sum_{l=0}^{n} \sum_{m=-l}^{l} C_{l}^{m}\left(\theta_{v}, \phi_{v}\right) H_{l}^{m}\left(\theta_{i}, \phi_{i}\right)+R_{n}
$$




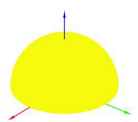

$\left|H_{0}^{0}\right|$

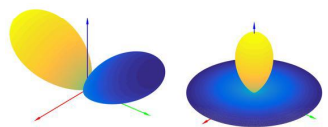

$\left|H_{1}^{-1}\right|$

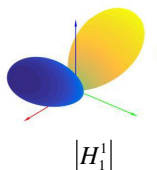

$\left|H_{1}^{\prime}\right|$

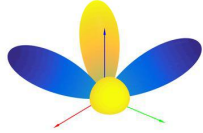

$\left|H_{2}^{-2}\right|$

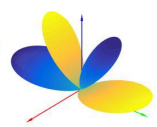

$\left|H_{2}^{-1}\right|$

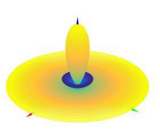

$\left|H_{2}^{0}\right|$

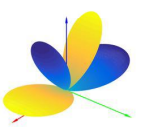

$\left|H_{2}^{1}\right|$

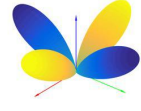

$\left|H_{2}^{2}\right|$

Figure 4. Function plots $H_{l}^{m}$ of the Hemispherical Harmonics basis for order $l$ from 0 to 2

where the coefficients $C_{l}^{m}$ can be obtained as the projection of $f$ onto each basis function (Eq. 9):

$$
C_{l}^{m}\left(\theta_{v}, \phi_{v}\right)=\int_{0}^{2 \pi} \int_{0}^{\frac{\pi}{2}} f\left(\theta_{v}, \phi_{v}, \theta_{i}, \phi_{i}\right) H_{l}^{m}\left(\theta_{i}, \phi_{i}\right) \sin \theta_{i} d \theta_{i} d \phi_{i}
$$

Visual representations of the first few real hemispherical harmonics are given Figure 4 where blue portions represent regions where $\left|H_{l}^{m}\right|$ is positive, and yellow portions represent where it is negative.

\section{DISCRETE MODAL DECOMPOSITION}

Resulting from vibratory mechanics, Discrete Modal Decomposition (DMD) models a geometrical element in a geometrical form space. DMD is applied in:

1. geometrical tolerancing ${ }^{\mathbf{2 3}, \mathbf{2 4}}$, to visualize the geometrical errors of surfaces and to specify the acceptable variations - form, position and orientation - of the real geometry around the target,

2. multiscale analysis of topographic surface roughness ${ }^{25,26}$, to characterize the form, waviness and roughness defects of a surface,

3. heat source evaluation ${ }^{\mathbf{2 7}, \mathbf{2 8}}$, to estimate the spatial term of a heat diffusion problem.

Similarly to the Discrete Fourier Transform or the Discrete Cosine Transform, this decomposition enables to make the projection of the measured surface into an eigen basis built from structural dynamics.

\subsection{Modal basis}

The hemispherical domain $\left[0, \frac{\pi}{2}\right] \times[0,2 \pi]$ designates the local hemisphere of BRDFs at surface points where the reflection integral is evaluated. An illuminated ideal diffuse reflecting surface will have equal luminance from all directions which lie in the half-space adjacent to the surface (Lambertian reflectance). The associated geometry (simple half dome in the proposed problem) leads to the definition of the dynamic structural problem which is classically defined as follows:

$$
M . \ddot{q}+K . q=0 \quad \text { with } \quad q=q(\theta, \phi)
$$

where $\mathrm{M}$ and $\mathrm{K}$ stand for the mass and the stiffness matrices respectively. Under such formalism, $q(\theta, \phi)$ stands for the displacements which characterizes the modal shapes defined as the harmonic solution of the equation 10. The eigen vectors $Q_{k}$ with the pulsations $w_{k}$ are solutions of the equation:

$$
q(\theta, \phi, t)=\sum_{k=1}^{+\infty} Q_{k} \cos \left(w_{k} t\right)
$$

and form the associated modal basis $\left(Q_{0}, Q_{1} \ldots, Q_{n-1}\right)$ where $\mathrm{n}$ is the number of modes used for the projection. The point of the present paper is to associate the displacement fields $q(\theta, \phi, t)$ to the measured luminances $L=\left(L_{0}, L_{1} \ldots, L_{N}\right)$ which draws the reflectance field. The eigen modes are determined by solving the linear system:

$$
\left(M^{-1} K-\frac{1}{w_{k}^{2}} I\right) Q_{k}=0
$$




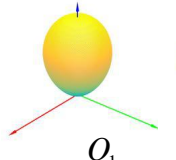

$Q_{1}$
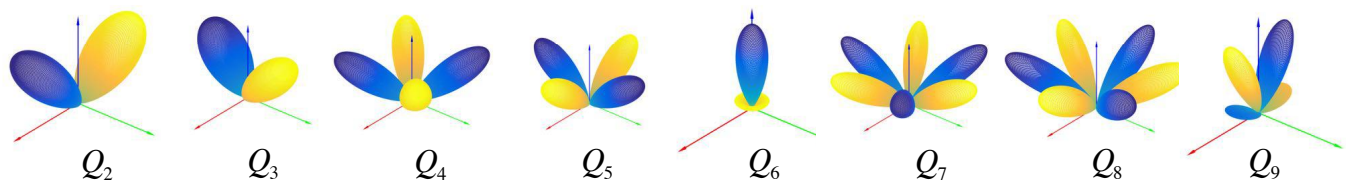

Figure 5. Function plots $Q_{k}$ of the Discrete Modal Decomposition basis (first 9 modes)

\subsection{Modelling}

Thus, any surface reflectance function $f$ can be expressed as the sum of a linear combination of the modal vectors and the decomposition residual $R_{n}$ (Eq.13):

$$
f_{\left(\theta_{v}, \phi_{v}\right)}\left(\theta_{i}, \phi_{i}\right)=\sum_{k=1}^{n} \lambda_{k}\left(\theta_{v}, \phi_{v}\right) Q_{k}\left(\theta_{i}, \phi_{i}\right)+R_{n}
$$

where the modal coefficients $\lambda_{k}$ can be obtained as the projection of the vector of measured luminances $L$ onto each modes of the non-orthonormal basis (Eq. 14):

$$
\lambda_{k}\left(\theta_{v}, \phi_{v}\right)=\left(Q_{k}^{T} Q_{k}\right)^{-1} Q_{k}^{T} \cdot L
$$

Thus, the modal parametrization uses a basis of adaptative number of modes to better represent surfaces. The first modal vectors composing the modal basis are given Figure 5 with the latter color represention of HSH descriptors. The complexity of shape and frequency increase when the order $n$ increases. If sampled at a high enough rate, the original function can in theory be perfectly reconstructed from the infinite set of samples. In practice, the number of modes used for the projection can not be equal to the number of measured luminances in order to avoid phenomenon known as aliasing or overfitting. So, the number of modes are chosen as to obey the Nyquist-Shannon sampling theorem.

\subsection{Rendering}

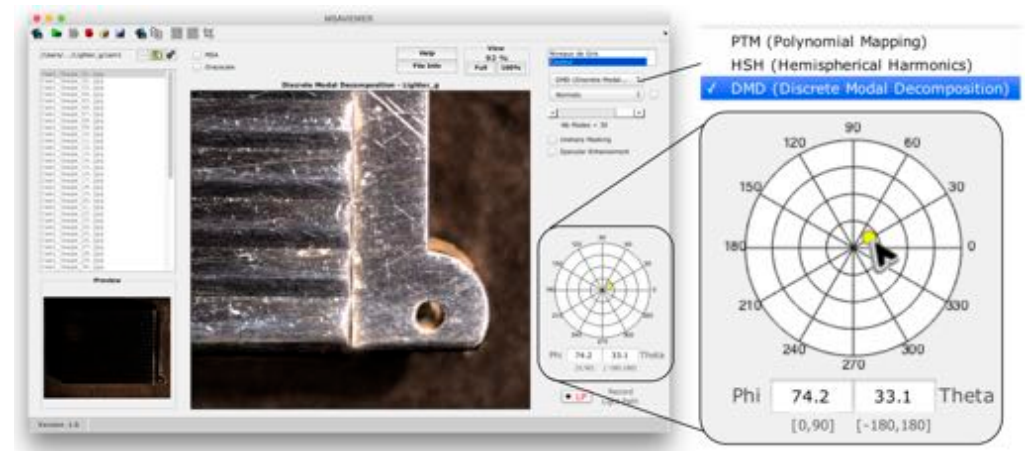

Figure 6. MSA-viewer interface. The user can control interactively the light direction by moving a mouse on an intuitive graphic in order to detect and evaluate particular aspects of surface appearance

A viewer (Figure 6) was implemented in Matlab including PTM, HSH and DMD reconstructions. The modal coefficients can be viewed as a modal amplitude spectrum that approximates the surface points color change as the lighting position changes. We established our RTI format in order to save the modal coefficients encoding the approximative reconstruction of the reflectance spectrum at each pixel in a computer file with the extension .msa (Measuring Surface Appearance). The MSA-viewer allows the controller to move the light in any direction and to aid him in detecting and evaluating particular aspects of surface appearance. 


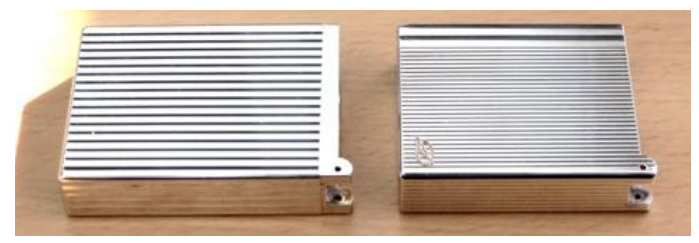

Figure 7. Two bodies of prestige ligther with guilloched pattern

\section{RESULTS}

\subsection{Application on high value-added industrial surfaces}

The RTI methods are applied to two surfaces of prestige lighter (Figure 7) in which a V-shaped pattern is engraved with an engine turning machine called guilloche to draw vertical lines on a surface at various spacing and widths. The surface can usually be achieved by light hand abrasion after depositing in a thin silver layer. Aspect defects have been identified on both surfaces, such as strockes and scratches that will be most apparent in the cropped views. After acquiring the sets of 56 images by our system, we compute the three approaches to obtain for each pixel the PTM, HSH and DMD contributions. Taking into account the sampling theorem, we use a modal basis composed of 32 modes, and according to the original embodiment 16 harmonics for HSH and 6 terms for PTM. We can interpolate and relight in the same light direction of the measured images in order to estimate the quality of RTI-based reconstructions whose results are presented in Section 4.2. Our comparative approach focuses on both the space of image representation and the space of lighting because RTI can be regarded as a mapping from the $4 \mathrm{D}$ space of lighting and viewing directions to the space of all $2 \mathrm{D}$ images.

\subsection{Comparison/discussion}

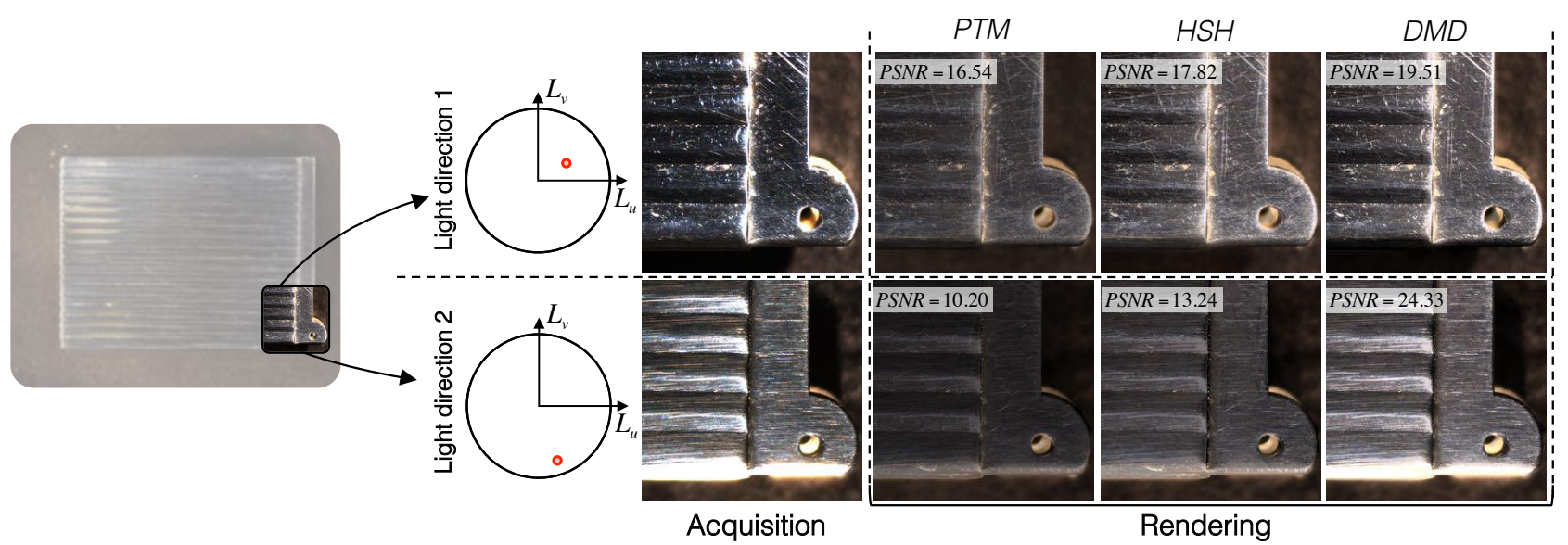

Figure 8. Cropped views of the original and reconstructed images of lighter 1 and their PSNR value in dB

Polynomial Texture Mapping removes the high frequencies of the reflectance field. The smoothing affects the light space, especially in regions where strong specular spikes (high-frequency) are observed (Figure 8). We compute the Peak Signal-to-Noise Ratio (PSNR) which is the most widely used objective image quality metric, in order to assess the quality of reconstruction in both light directions of the device that are chosen arbitrarily. The higher the PSNR, the better the quality of the reconstructed image. Even if the PSNR value indicates the higher quality for DMD reconstruction, it's important to recognize its limitations:

- it relies strictly on numeric comparison based on a pixel-by-pixel comparison of images and does not actually take into account either the location of observable reconstruction degradation nor any level of biological factors of the human vision system, 
- it does not provide information along the light space of the RTI techniques. PTM technique tends to transform the measured luminances distribution in the diffuse (matte) surfaces (gaussian distribution) while DMD and, to a lesser extent, HSH preserve the shapes of luminance distributions as illustrated in Figure 3.

A consequence of this transformation of luminance distributions can be observed in Figure 9. Our perceptual assessment differs between the image obtained with the light direction 1 and 2. However, changing lighting direction does not much affect the visual appearance of the surface in rendered images produced with the HSH and PTM fitters. DMD can account much luminance variation, for example in low-angled light, the stroke appears as a clear spot in a dark zone like on the original image. Sudden and strong changes in the constrast can automatically draw visual attention to their locations when the user moves the virtual light in any direction. When the controller has to evaluate the criticity of a detected anomaly, he automatically moves the light source to yield local and global processing of contrast variations.

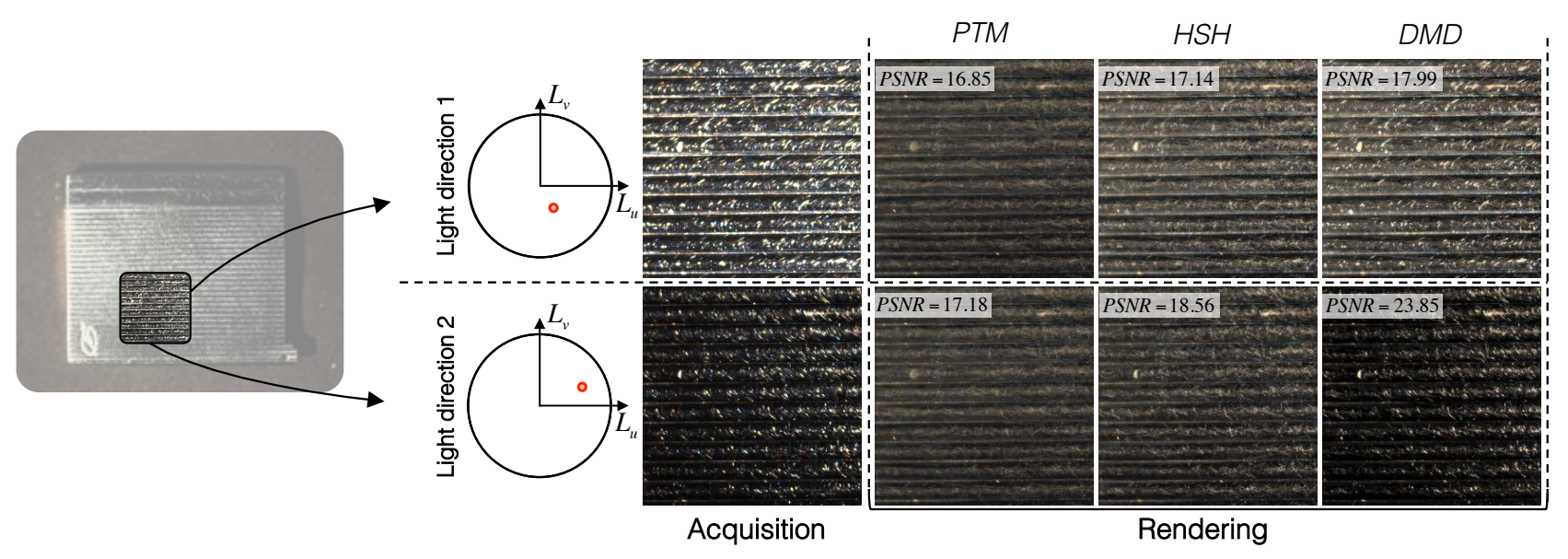

Figure 9. Cropped views of the original and reconstructed images of lighter 2 and their PSNR value in dB

\section{CONCLUSIONS AND PERSPECTIVES}

This paper presents a robust and effective approach to model the local reflectance from the photometric RTI data acquisitions, basing on a parameterization named Discrete Modal Decomposition. By varying the illumination position, the RTI technique aims at enriching the classical information conveyed by images. Each pixel is described by a set of values rather than one value classically; each value corresponding to a specific illumination position. The local reflectance of each pixel can thus be modelled from the set of luminance values associated to each illumination direction. This modelisation is generally performed by applying an interpolation (or approximation) of the discrete acquired data, using a continuous family of functions to describe each pixel local reflectance. Then, this simplified model allows the surface to be virtually rendered for any (chosen) illumination incidence (azimuth and elevation), and the surface can therefore be inspected by varying virtually the direction of illumination. This exploration enhances the micro-geometry and roughness local morphology, and for industrial applications, allows appearance surace quality and perception to be better controlled.

The relevance of the DMD approach is investigated by comparing the results with the ones obtained performing the two most used RTI methods, which are respectively associated to a second order polynomial (PTM) and an hemispherical harmonics (HSH) vector basis. Particularly, this study figures out an interesting feature of the DMD-based reflectance modelisation, that consists in its ability to describe complex local reflectance in terms of angular and intensity luminance variations. If similar results are obtained for lambertzian diffuse reflectances, a more reliable approximation of specular lobes, and more generally of glossy surface local areas is provided by this approach. 
Future work will include an assessment of RTI reconstruction quality by analyzing more precisely how this new technique behaves, and particularly, the evolution of luminance local distribution from the acquisition to the RTI modelisation/reconstruction will be investigated. The evaluation of the RTI methods will be carry out statistically in order to better quantify their relevance.

\section{REFERENCES}

1. F. E. Nicodemus, "Directional Reflectance and Emissivity of an Opaque Surface," Applied Optics 4, pp. 1-8, 1965.

2. F. E. Nicodemus, J. C. Richmond, J. J. Hsia, I. W. Ginsberg, and T. Limperis, Geometrical considerations and nomenclature for Reflectance, Institute for Basic Standards, National Bureau of Standards, Washington, 1977.

3. J. J. Koenderink, A. J. van Doorn, and M. Stavridi, "Bidirectional Reflection Distribution Function expressed in terms of surface scattering modes," Computer Vision-ECCV'94 , pp. 28-39, 1996.

4. J. F. Murray-Coleman and A. M. Smith, "The automated measurement of BRDFs and their application to luminaire modeling," Journal of the Illuminating Engineering Society 19, pp. 87-99, 1990.

5. K. J. Dana, B. van Ginneken, S. K. Nayar, and J. J. Koenderink, "Reflectance and Texture of Real-World Surfaces," ACM Transactions on Graphics 18, pp. 1-34, 1999.

6. G. Ged, G. Obein, Z. Silvestri, J. Le Rohellec, and F. Vienot, "Recognizing real materials from their glossy appearance," Journal of Vision 10, pp. 18-18, July 2010.

7. L. W. MacDonald, "Visualising an Egyptian Artefact in 3D: Comparing RTI with Laser Scanning," Proceedings of the 2011 international conference on Electronic Visualisation and the Arts EVA'11, pp. 155-162, 2011.

8. M. Zhang and M. S. Drew, "Efficient robust image interpolation and surface properties using polynomial texture mapping," Eurasip Journal on Image and Video Processing, pp. 1-36, 2014.

9. J. D. Durou, "Shape from shading - Eclairages, réflexions et perspectives," tech. rep., 2007.

10. T. Malzbender, D. Gelb, and H. Wolters, "Polynomial texture maps," Proceedings of the 28th annual conference on Computer graphics and interactive techniques, pp. 519-528, 2001.

11. T. Malzbender and D. G. Gelb, "Apparatus for and method of enhancing shape perception with parametric texture maps," 2000.

12. H. J. Wolters, "Apparatus for and method of converting height fields into parametric texture maps," (6583790), 2000.

13. T. Malzbender, "Direction-dependent texture maps in a graphics system," (6297834), 2001.

14. B. A. Ritter, "System and method for synthesis of parametric texture map textures," 2004.

15. T. Malzbender and D. G. Gelb, "Apparatus for and method of rendering 3D objects with parametric texture maps," Oct. 2005.

16. N. Horton and B. A. Ritter, "System and method for combining parametric texture maps," (7009620), 2006.

17. S. H. Westin, J. R. Arvo, and K. E. Torrance, Predicting Reflectance Functions from Complex Surfaces. $\mathrm{PhD}$ thesis, Faculty of the Graduate School of Cornell University, 1992.

18. P. Gautron, J. Krivanek, S. N. Pattanaik, and K. Bouatouch, "A Novel Hemispherical Basis for Accurate and Efficient Rendering," Eurographics Symposium on Rendering 2004, pp. 1-10, 2004.

19. J. Kautz, P. P. Sloan, and J. Snyder, "Fast, arbitrary BRDF shading for low-frequency lighting using spherical harmonics," in Proceedings of the 13th Eurographics Workshop on Rendering, 2002.

20. M. Mudge, T. Malzbender, A. Chalmers, and R. Scopigno, "Image-based empirical information acquisition, scientific reliability, and long-term digital preservation for the natural sciences and cultural heritage," homepages.inf.ed.ac.uk, 2008.

21. J. Krivanek, J. A. Ferwerda, and K. Bala, "Effects of global illumination approximations on material appearance," ACM Transactions on Graphics (TOG) 29(4), pp. 1-10, 2010.

22. G. Palma, M. Corsini, and P. Cignoni, "Dynamic shading enhancement for reflectance transformation imaging," Journal on Computing and Cultural Heritage 3, 2010.

23. H. Favreliere, Modal Tolerancing : From metrology to specifications. PhD thesis, Université de Savoie, 2009. 
24. S. Samper, P.-A. Adragna, H. Favreliere, and M. Pillet, "Modeling of 2D and 3D Assemblies Taking Into Account Form Errors of Plane Surfaces," Journal of Computing and Information Science in Engineering $\mathbf{9}$, p. 041005, Dec. 2009.

25. G. Le Goic, H. Favreliere, S. Samper, and F. Formosa, "Multi scale modal decomposition of primary form, waviness and roughness of surfaces," Scanning, the journal of scanning microscopies - Special Issue on Surface Metrology II 33(5), pp. 332-341, 2011.

26. J. Grandjean, G. Le Goic, H. Favreliere, Y. Ledoux, S. Samper, F. Formosa, L. Devun, and T. Gradel, "Multi-scalar analysis of hip implant components using modal decomposition," Measurement Science and Technology 23(12), 2012.

27. T. Pottier, H. Louche, S. Samper, H. Favreliere, F. Toussaint, and P. Vacher, "A new filtering approach dedicated to heat sources computation from thermal field measurements," PhotoMechanics Conference, Montpellier : France, pp. 1-4, 2013.

28. T. Pottier, H. Louche, S. Samper, H. Favreliere, F. Toussaint, and P. Vacher, "Proposition of a modal filtering method to enhance heat source computation within heterogeneous thermomechanical problems," International Journal of Engineering Science, pp. 1-14, 2014. 